\title{
An Iterative Atmospheric Phase Screen Compensation for Near-Real-time Ground-Based InSAR Measurements Over a Mountainous slope
}

\author{
Yuta Izumi, student member, IEEE, Lilong Zou, Member, IEEE, Kazutaka Kikuta, Member, IEEE, \\ and Motoyuki Sato, Fellow, IEEE
}

\begin{abstract}
In this paper, an atmospheric phase screen (APS) compensation algorithm for a near real-time Ground-Based InSAR (GB-InSAR) over a mountainous area is investigated. A novel APS compensation scheme is proposed to compensate the fluctuated APS caused by a spatial 3D inhomogeneous refractivity index distribution without any a priori knowledge of moving location. The proposed method simultaneously addresses to identify moving pixels by a criterion of absolute velocity estimated by the Coherent Pixels Technique (CPT). The proposed method consists mainly of three steps; (1) the stratified APS compensation, (2) identification of moving pixel candidate, and (3) the residual APS (remained APS after (1)) compensation by Kriging interpolation. The steps mentioned above are iteratively applied in order to increase the accuracy of the whole process. In this framework, we develop the 2D quadratic polynomial model of the refractivity index with respect to slant range and topographic height for modeling the stratified APS. Furthermore, a prediction of the residual APS is achieved by applying the IRF- $k$ Kriging interpolation, taking into account the non-stationarity of the residual APS. We evaluate the proposed method using zerobaseline GB-DInSAR data over a mountainous area located in Minami-Aso, Kumamoto, Japan through the near real-time postlandslide measurement campaign.
\end{abstract}

Index Terms-Atmospheric phase screen, GB-SAR, DInSAR

\section{INTRODUCTION}

Grov round-based synthetic aperture radar (GB-SAR) has increasingly been used as a powerful remote sensing tool for environmental monitoring [1], [2]. With an advantage of temporally dense measurement compared to spaceborne or airborne SAR, differential SAR interferometry (DInSAR) applied to GB-SAR provides detail time-series information of land displacement. Besides, one of the benefits of GB-SAR is

${ }^{\mathrm{T}}$ his paragraph of the first footnote will contain the date on which you submitted your paper for review. It will also contain support information, including sponsor and financial support acknowledgment. For example, "This work was supported in part by the U.S. Department of Commerce under Grant BS123456."

The next few paragraphs should contain the authors' current affiliations, including current address and e-mail. For example, F. A. Author is with the National Institute of Standards and Technology, Boulder, CO 80305 USA (email: author@ boulder.nist.gov). recognized as its zero-baseline configuration as the preferred configuration of minimizing decorrelation sources on the interferometric phase.

Although plenty of decorrelation sources are avoidable in zero-baseline GB-SAR configuration, the reflectivity change between the interferometric combination of two radar images incurs the strong atmospheric artifacts providing the phase delays, referred to as atmospheric phase screen (APS). The APS is the most relevant disturbance for a GB-SAR measurement, and it might eliminate a spatial signature of actual land deformation. Especially, when we work on near real-time monitoring for hazard anticipation of a steep mountainous slope, strong APS effects lead to a false alarm of an early-warning system. Accordingly, its compensation is highly required as post-processing to provide the correct displacement value.

Some compensation approaches have been proposed for the GB-SAR application [3]-[11]. Most of the literature concentrates on the data-driven model-based statistical technique without any a priori meteorological information [4][6] whereas the method in [7] proposed to use on-site meteorological information to calibrate humidity-based APS model. Under the assumption of homogeneous refractivity cases such as the soft topography area, a model-based solution assumes the linear relationship between APS and the position in the range [4], [5]. In such a case, an estimated linear phaseramp is subtracted from the measured DInSAR image to achieve APS-free results. On the other hand, a steep topographic area does not hold a homogeneous assumption of the refractivity over the observation area because of vertically stratified refractivity. In [6], the multiple regression model (MRM) was applied to compensate for the topography correlated APS (vertically stratified APS) in a mountainous slope using the external digital elevation model (DEM)

Y. Izumi is with the Graduate School of Environmental Studies, Tohoku University, Sendai, Japan (e-mail: yuta.izumi.r8@dc.tohoku.ac.jp).

L. Zou is with the School of Computing and Engineering, University of West London, London, W6 5RF U.K. (e-mail: Lilong.Zou@uwl.ac.uk).

K. Kikuta is with Center for Northeast Asian Studies, Tohoku University, Sendai, Japan (e-mail: kazutaka.kikuta.e6@tohoku.ac.jp).

M. Sato is with Center for Northeast Asian Studies, Tohoku University, Sendai, Japan (e-mail: motoyuki.sato.b3@tohoku.ac.jp). 
information, referred to APS-MRM. By constraining the small scenario, the linear relationship between the refractivity index and topographic height is assumed in the MRM, leading to a quadratic model of the stratified APS.

When we work on a mountainous slope measurement, the stratified APS is properly modeled by the APS-MRM. However, in the presence of a spatial 3D inhomogeneity of the refractivity distribution, the residual component of the APS after the stratified APS compensation must be taken into account. Furthermore, a simple linear relationship between a topographic height and a refractivity in the APS-MRM is no longer valid. In this case, the APS $\phi_{\text {APS }}$ presented within the troposphere can be expressed by mixing of the stratified APS $\phi_{\text {str }}$ and the residual APS $\phi_{\text {res }}$, where the $\phi_{\text {res }}$ includes a longer scale component $\phi_{\text {long }}$ due to the horizontal variation of refractivity and a short-scale component $\phi_{\text {short }}$ [12]-[15], as

$$
\phi_{\mathrm{APS}}=\phi_{\mathrm{str}}+\phi_{\text {res }}=\phi_{\text {str }}+\phi_{\text {long }}+\phi_{\text {short }}
$$

In spaceborne SAR applications, Numerical Weather Prediction (NWP) models have been proposed to model propagation delay in the atmosphere [16], [17] with the larger spatial scale, which is not easily applied to GB-SAR. Besides, the time-series InSAR analysis with a certain number of SAR images is able to mitigate the residual APS by means of spatiotemporal properties. A number of time-series InSAR approaches have been proposed [18]-[21], pioneered by the Permanent Scatterer Interferometry (PSI) [18], [19]. The PSI aims to identify the permanent scatterer (PS) pixels which are point-wise scatterers. Those advanced differential InSAR (DInSAR) algorithms treat the APS as spatially low frequency and temporally high-frequency signal [18], [20]-[22] relative to the deformation signal. Hence a spatial low-pass and temporal high-pass filtering possibly isolate APS from deformation signal if there is no seasonal trend and stratified APS which are the temporally correlated terms [23]. This spatiotemporal assumption is reasonable for the spaceborne SAR application since its acquisition interval is in the order of days. However, the APS is temporally correlated in the operation of near realtime GB-SAR [24], [25] because the acquisition interval is usually in the order of minutes. Therefore, in this particular case, a direct adaptation of conventional advanced DInSAR algorithm is not feasible. Accordingly, a modification is required for near-real-time GB-SAR measurements.

Besides, the geostatistics theory has been used to stochastically model the uncertain spatial variation to be used for linear minimum-variance unbiased estimation, also known as the Kriging [26]. The spatial or temporal prediction is thus achieved by a weighted average of a linear combination of the observed data. Precise analysis of the structural characteristic by the geostatistical approach is thus appropriate for modeling of the short-scale APS component based on the elementary Kolmogorov turbulence theory [27]. Therefore, the analysis and inference of the structure-function and the covariance are essential to mitigate the fluctuated APS [15], [28]. Under the second-order stationarity assumption, the covariance function is inferred by a theoretical variogram to model the uncertain spatial variation [29]. However, if the moving pixels are involved in the covariance inference and the linear combination

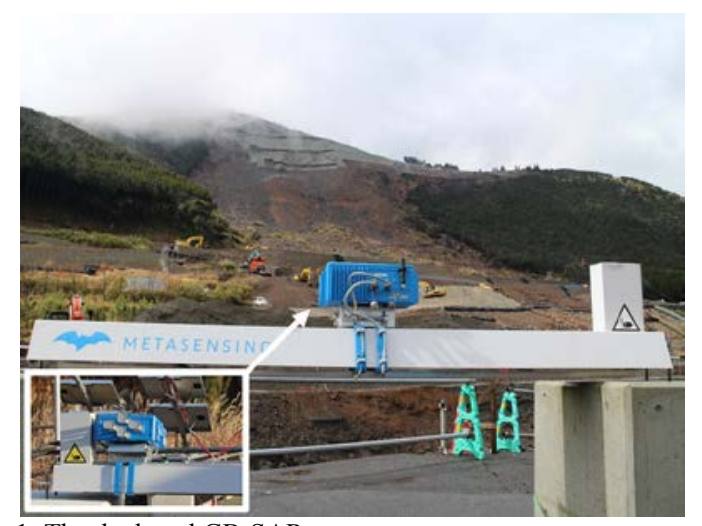

Fig.1. The deployed GB-SAR system.

of the observed data to be used in the weighted averaging process, a spatial prediction leads to an ill-posedness. Nonetheless, an extrapolation of the APS from known motionless points by the Kriging has actually been done with $a$ priori knowledge of moving areas such as glacier measurement in [15].

In those backgrounds, a data-driven APS compensation method without any a priori knowledge of moving location for GB-SAR measurements is investigated in this paper. To achieve it, we herein propose a novel iterative APS compensation scheme incorporating the atmospheric disturbances shown in (1). The idea of the method is to iteratively identify and mask the moving pixels and perform the spatial extrapolation over the identified pixels. In this framework, the following contributions are involved in the novel compensation scheme;

1) The $2 \mathrm{D}$ quadratic polynomial function of the refractivity index with respect to a slant range and a topographic height is introduced to be used for a stratified APS model.

2) The intrinsic random function of order $k$ (IRF- $k$ ) Kriging is introduced to extrapolate the residual APS.

3) An iterative algorithm based on the advanced DInSAR algorighm is proposed to compensate the APS without any a priori knowledge of moving location.

The proposed method is validated in a mountainous area. We deployed Ku-band FMCW GB-SAR as a near-real-time early warning system over the landslide affected slope in MinamiAso, Kumamoto, Japan. The presence of an anomalous refractivity distribution in our target area is experimentally validated by the in-situ meteorological measurement of weather stations in two locations. This observation assists to claim the necessity of modification of the conventional APS compensation technique for GB-SAR.

\section{A GB-SAR CAMPAIGN AND A MESOSCALE ATMOSPHERIC CONDITION}

\section{A. An overview of the GB-SAR campaign}

Starting from the foreshock occurred on 14th April 2016, a series of earthquakes hit Kumamoto prefecture in the Kyusyu region of Japan [23]. As a consequence, at least 97 landslides were found, which mainly concentrated in the Aso caldera. The 
most noticeable landslide has occurred in Minami-Aso located
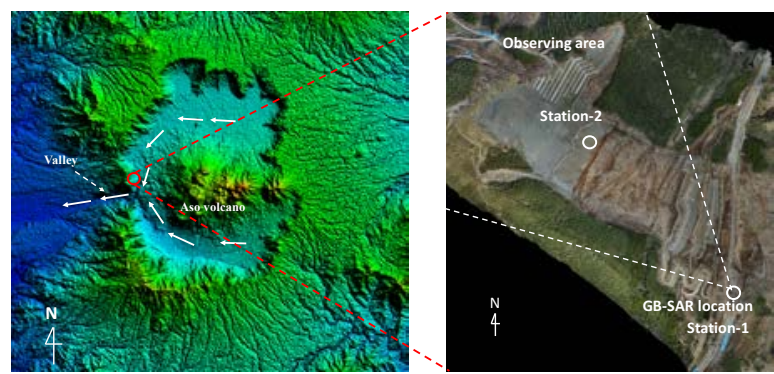

Fig. 2. The DEM image of the Aso caldera with the Aso volcano cone in the center and the location of two installed weather stations. A red circle indicates the GB-SAR observation area located in the outer rim of the caldera. The white arrows represent nocturnal wind direction.

in the outer rim of the caldera destroyed a $200 \mathrm{~m}$ Aso large bridge. A disaster restoration project was launched in order to reconstruct the damaged road and reinforce the landslide affected slope. In such a case, the unstable zone whose potential of post-landslide threatens the on-site operator. Under this situation, a GB-SAR system is deployed for risk mitigation from March 2017 up to now. Owing to its continuous operation mode [1], the deployed GB-SAR has the potential to issue an early warning in near-real-time.

The GB-SAR system used in this study is an FMCW radar system, which allows the fast sampling rate with $5 \mathrm{sec}$ acquisition time shown in Fig. 1, developed by METASENSING [16]. This fast acquisition capability circumvents the anomalous APS caused by the refractivity change during aperture synthesis [24]. The system operates in $\mathrm{Ku}$-band with a center frequency of $17.2 \mathrm{GHz}$ and a frequency bandwidth of $300 \mathrm{MHz}$ and has the capability of fully polarimetric acquisition.

\section{B. An atmospheric condition of the observation area}

The digital elevation model (DEM) of the Aso caldera is displayed in Fig. 2. As we can see, the Aso-caldera consists of the large-scale caldera with Aso volcano cone in the center, known as the double volcano. This landform consists of the basin between the outer rim of the caldera and the volcano cone. A unique cut line of the west part of the outer rim, which is a thin valley plays an important role in draining the water from the caldera.

The atmospheric condition on the test area is drastically variated in time and space due to the local wind variations. The general mesoscale meteorological condition in the basin region proves a significant difference in a refractivity distribution between day-time and night-time. In order to investigate temporal meteorological behavior in our area, two weather stations are employed. In Fig. 2, locations of two weather stations are illustrated. The line of sight distance and relative height between two stations are $500 \mathrm{~m}$ and $115 \mathrm{~m}$, respectively. One of the weather stations is installed just behind the GB-SAR location (station-1) and another weather station was installed in the middle of the slope (station-2). In this configuration, the temporal atmospheric parameters of different locations can be investigated. Two weather stations collected three-days data from 19:00 May 28th until 11:50 May 30th, 2019, with a time separation of $15 \mathrm{~min}$. Note that there is no precipitation during

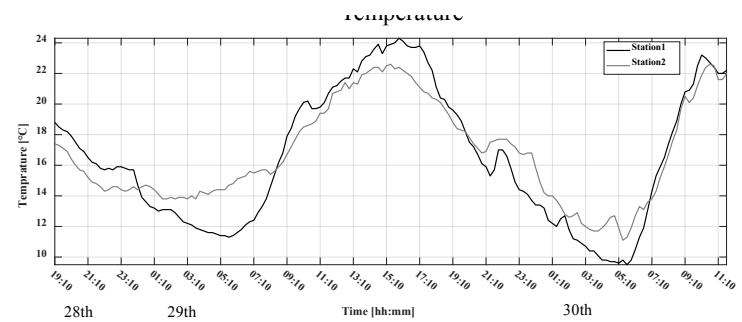

(a)

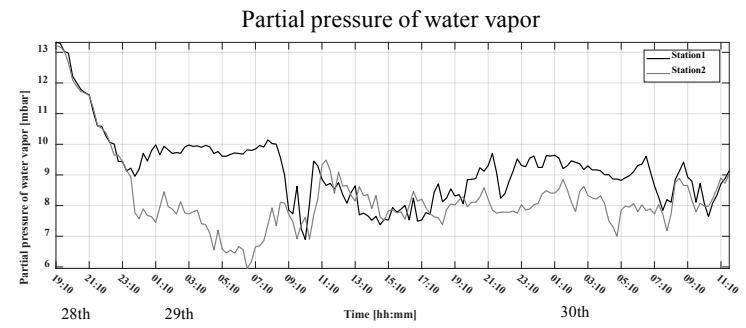

(b)

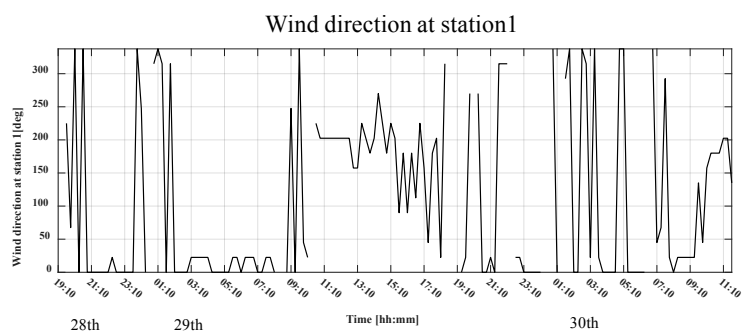

(c)

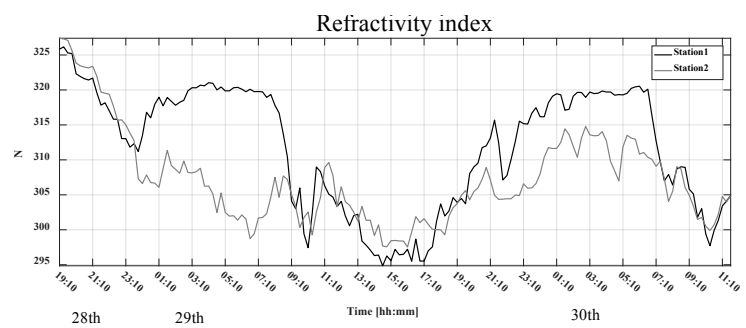

(d)

Fig. 3. The temporal meteorological data collected by two weather stations with $15 \mathrm{~min}$ time interval. (a) Temperature; (b) partial pressure of water vapor; (c) wind direction at station-1; (d) refractivity index. Black and gray solid lines show measured data by the station- 1 and the station-2, respectively.

meteorological measurements. The measured time-series results of temperature and partial pressure of water vapor which are the parameters of the refractivity index are shown in Fig. 3(a) and (b), respectively. The wind direction (0deg is set as north wind direction) measured in station-1 is shown in Fig. 3(c). Furthermore, the refractivity index computed [30] through the measured atmospheric parameters is shown in Fig. 3(d).

In the night time after sunset, radiative cooling of a mountainous slope makes overlying air cold and dense. In turn, the dense air sinks along the slope surface to the basin bottom, leading to a cold air accumulation [31]. This downward wind is often termed as the katabatic wind. As a result of the cold air pool in the basin floor, the temperature in the basin becomes much lower than the higher altitude slope, which is called temperature inversion. The observed temperature in Fig. 3(a) 
indicates this temperature inversion at midnight where the station-1 shows a lower value than the station- 2 with $\sim 3^{\circ} \mathrm{C}$ difference in maximum. Besides, the unique wind process is made in the Aso caldera basin where the cold air formed in the Aso basin flows out from the valley in the west part of outer rim due to the pressure difference between inside and outside of caldera [32], indicated by white arrows in Fig. 2. The measured wind direction at station-1 shown in Fig. 3(c) shows this cold air wind, which indicates north wind direction ( $0 \mathrm{deg}$ ) during a temperature inversion. This winding process possibly advects the air parcel from different locations. The partial pressure of water vapor between both stations shows a significant difference during a temperature inversion, which may be due to advection of high moist air by cold airflow in the basin floor, shown in Fig. 3(b).

While after sunrise, the heated air in the basin is positively buoyant and starts to rise due to the insolation. This causes air circulation in the convective boundary layer [33]. This circulation process effectively transports the heated air over the slope to the entire basin, resulting in the breakup of temperature inversion formed during the night [34]. The breakup of temperature is shown in around 29th 08:10 in Fig. 3(a). Consequently, the atmospheric condition after the sunset is hashed by this local wind process.

The advection flow by several local winds reveals the high variability between a nocturnal and diurnal atmospheric condition. Accordingly, atmospheric parameters such as temperature and water vapor are variated on a site-to-site atmospheric condition regardless of topographic height. As a result, the refractivity index in Fig. 3(d) shows a high temporal variation too, which is accordant temporal behavior with water vapor in Fig. 3(b).

\section{APS COMPENSATION PROCEDURE}

The APS compensation procedure consists of three steps, which can be simply summarized as follows;

1) The stratified APS compensation (Section-III A)

2) Identification of moving pixel candidate (Section-III C)

3) The residual APS compensation (Section-III B)

The first step dedicates to compensate the APS with respect to slant range and topographic height while the third step takes into account the remained APS. The critical part of the proposed method is involved in the second step which is discrimination between moving pixels and non-moving pixels. The above steps are iteratively applied in order to increase the accuracy of the whole process. The overall compensation procedure is explicitly demonstrated in Section III C.

\section{A. Stratified APS modeling and compensation}

The two-way propagation atmospheric phase term $\varphi_{\text {atm }}$, backscattered from slant range distance $r_{s}$ can be expressed by the refractivity index $N\left(r_{s}, t\right)$ which is a spatiotemporal function of the temperature $T(\mathrm{~K})$, the pressure $P$ (in millibars), and the partial pressure of water vapor at the slant range $r_{s}$ and the time $t$, as

$$
\varphi_{\text {atm }}(t)=10^{-6} \frac{4 \pi f_{c}}{c} \int_{l} N\left(r_{s}, t\right) d r_{s},
$$

where $f_{c}$ is the center frequency. The stratified APS can be obtained by taking a difference of the atmospheric phase as a function of a temporal separation $T$ between time $t_{1}$ and $t_{2}$.

$$
\phi_{\mathrm{APS}}(T)=\varphi_{\mathrm{atm}}\left(t_{2}\right)-\varphi_{\mathrm{atm}}\left(t_{1}\right),
$$

In a steep topographic scenario, the $N$ is assumed to be related to a topographic height $z$ by an exponential profile [35]

$$
N(z)=N^{s} e^{-\alpha z},
$$

where $N^{s}$ is the refractivity index at the sea-level, $z$ is the height above the sea-level, and $\alpha$ accounts for the decay parameter. Based on the in-situ meteorological measurements, Iglesias et al. reported that the $N$ indicates a linear behavior with a topographic height [6]. In this experimental background, the $N$ in (4) is further approximated by the first two terms of the Taylor series, as

$$
N(z)=N^{s}-N^{s} \alpha z,
$$

Since (5) states a linear behavior with respect to the topographic height $z$, the differential refractivity index $\Delta N$ also becomes a linear function with respect to $z$.

By substituting (5) into (2) and performing (3), the unwrapped stratified APS can be obtained as

$$
\begin{gathered}
\phi_{\mathrm{str}}^{\text {linearN }}\left(t_{1}, t_{2}\right)=10^{-6} \frac{4 \pi f_{c}}{c}\left(\Delta N^{s} r_{s}-\Delta N^{s} \alpha \frac{r_{s} z_{d}}{2}\right) \\
=\beta_{1}^{\prime} \cdot r_{s}+\beta_{2}^{\prime} \cdot z_{d} \cdot r_{s},
\end{gathered}
$$

where $\Delta N^{s}=N^{s}\left(t_{2}\right)-N^{s}\left(t_{1}\right)$, and $z_{d}$ is the height above the GB-SAR location. The unknown coefficients $\beta_{1}^{\prime}$ and $\beta_{2}^{\prime}$ are solved by the least-squares regression through the MRM as in [6].

Because of a 3D spatial heterogeneity of atmospheric parameters presented over the mountainous slope, a distribution of the refractivity index becomes spatially heterogeneous too. Especially, the in-situ measurement demonstrated a spatial variation of meteorological parameters. In this case, the assumed linear function of the refractivity index with respect to a topographic height must be more generalized for estimating a global trend of the APS along the slant range axis. Here we model the refractivity index as a quadratic $2 \mathrm{D}$ polynomial function with respect to a topographic height $z$ and a slant range $r$ as

$$
\begin{aligned}
& N^{2 \text { Dpoly }}(z, r) \\
& =N_{0}^{\text {poly }}+N_{1}^{\text {poly }} \cdot z+N_{2}^{\text {poly }} \cdot z^{2}+N_{3}^{\text {poly }} \cdot z+N_{4}^{\text {poly }} \cdot z^{2}+N_{5}^{\text {poly }} z \cdot r
\end{aligned}
$$

where $N_{\eta}^{\text {poly }}(\eta=0,1,2,3,4$, and 5) represents the coefficients of the polynomial. Notice that the refractivity index in (7) is no longer a linear function with respect to height. By substituting (7) into (2) and performing (3), the unwrapped stratified APS can be obtained as 


$$
\begin{aligned}
& \phi_{\text {str }}^{\text {poly }}\left(t_{1}, t_{2}\right) \\
& =10^{-6} \frac{4 \pi f_{c}}{c}\left(\Delta N_{0}^{\text {poly }} r_{s}+\Delta N_{1}^{\text {poly }} \frac{r_{s} z_{d}}{2}+\Delta N_{2}^{\text {poly }} \frac{r_{s} z_{d}{ }^{2}}{3}\right. \\
& \left.+\Delta N_{3}^{\text {poly }} \frac{r_{s}^{2}}{2}+\Delta N_{4}^{\text {poly }} \frac{r_{s}^{3}}{3}+\Delta N_{5}^{\text {poly }} \frac{r_{s}^{2} z_{d}}{3}\right) \\
& =\beta_{0} r_{s}+\beta_{1} r_{s} z_{d}+\beta_{2} r_{s} z_{d}^{2}+\beta_{3} r_{s}^{2}+\beta_{4} r_{s}^{3}+\beta_{5} r_{s} z_{d}
\end{aligned}
$$

The (8) states the stratified APS model based on the 2D polynomial function $N^{2 \mathrm{Dpoly}}$ in (7). The LS regression is then performed to estimate the $\beta$ similarly as the APS-MRM method [6]. It is important to note that the more degree of the polynomial model in (7) increases, the more flexible fitting for the APS is achieved. However, an improper expansion will lead to an ill-fitted problem and may remove the local spatial signature which is a real displacement signal. Therefore, the stratified APS must be performed to compensate for only a global trend.

\section{B. Residual APS prediction in space}

In the theory of a random function (RF), a residual differential phase $\phi_{\text {res }}$ is considered to be the realization of a $\mathrm{RF} Z(\mathbf{x})$ of the spatial coordinate $\mathbf{x} . \phi_{\text {res }}$ is further decomposed into two terms; longer scale component $\phi_{\text {long }}(\mathbf{x})$ and a shortscale component $\phi_{\text {short }}(\mathbf{x})$ as shown in (1). Due to the presence of slowly varying deterministic function $\phi_{\text {long }}(\mathbf{x})$ known as drift, the mean of a RF is no more constant and varied slowly in space, indicating the non-stationarity of the RF and no finite variance, as commented by [12]. In this case, the inference of the covariance function is not straightforward because the covariance is defined under the second-order stationarity. Accordingly, the approach to infer the semivariogram of $\phi_{\text {short }}(\mathbf{x})$ (i.e., underlying semivariogram) in the presence of $\phi_{\text {long }}(\mathbf{x})$ must be taken into account. In the case of the nonstationarity, mainly two geostatistical methods are found; universal Kriging and intrinsic random function of order $k$ (IRFk) Kriging. Because the universal Kriging process falls into a circular problem where the drift form should be known in the Kriging system although the drift form should be estimated through the universal Kriging system, we herein apply the IRF$k$ Kriging to predict $\phi_{\text {res }}$. The IRF- $k$ Kriging is introduced for the simultaneous estimation of the drift model and the covariance function [28], [36]. In this method, the drift and the covariance are decomposed through increments of a sufficient order to filter out the drift and achieve stationarity [26].

In IRF- $k, \phi_{\text {res }}$ turns to be a local model of the form

$$
\begin{aligned}
\phi_{\text {res }}(\mathbf{x}) & =\phi_{\text {long }}(\mathbf{x})+\phi_{\text {short }}(\mathbf{x}) \\
& =\sum_{l=0}^{k} a_{l} f^{l}(\mathbf{x})+\phi_{\text {short }}(\mathbf{x})
\end{aligned}
$$

where $\alpha_{l}$ represents the unknown coefficients and $f^{t}$ is known basis functions of spatial coordinates, (i.e., $f^{1}=x, f^{2}=y, f^{3}=x^{2}$, $f^{4}=y^{2}, f^{\beta}=x y$ ). Considering a set of weights $w_{\alpha}$ applied to points $\mathbf{x}_{\alpha}$, a linear combination of these weights with $n$ random variables at locations $\mathbf{x}_{\alpha}$

$$
\phi_{\text {res }}(\mathbf{w})=\sum_{\alpha=1}^{n} w_{\alpha} \phi_{\text {res }}\left(\mathbf{w}_{\alpha}\right),
$$

is called to be an allowable linear combination of order $k$ (ALC$k$ ) or generalized increment of order $k$, if following conditions are satisfied,

$$
\sum_{\alpha=1}^{n} w_{\alpha} f^{l}\left(\mathbf{x}_{\alpha}\right)=f^{l}\left(\mathbf{x}_{0}\right)
$$

which hold for all monomials of order $\leq k$. By introducing $w_{0}=-1$ into (11), the following condition is given

$$
\sum_{\alpha=0}^{n} w_{\alpha} f^{l}\left(\mathbf{x}_{\alpha}\right)=0
$$

This equation explicitly indicates the filtering effect of $f^{t}(\mathbf{x})$ with respect to the set of points $\mathbf{X}_{\alpha}$. If the linear combination $\sum_{\alpha=1}^{n} w_{\alpha} \phi_{\text {res }}\left(\mathbf{x}_{\alpha}+\mathbf{h}\right)$ is second-order stationarity whatever the ALC- $k w$, its RF is called IRF- $k$.

The covariance of generalized increment of order $k$ is denoted by $K(\mathbf{h})$ and used to describe the correlation structure, namely generalized covariance (GC);

$$
\operatorname{Var}\left(\sum_{\alpha=1}^{n} w_{\alpha} \phi_{\mathrm{res}}\left(\mathbf{x}_{\alpha}\right)\right)=\sum_{\alpha=1}^{n} \sum_{\beta=1}^{n} w_{\alpha} w_{\beta} K\left(\mathbf{x}_{\alpha}-\mathbf{x}_{\beta}\right)
$$

One challenging step in the IRF- $k$ Kriging is the inference of the GC. In practice, the GC is modeled as the polynomial GC function [26], [37]

$$
K(\mathbf{h})=C_{0} \delta(\mathbf{h})+\sum_{i=0}^{m} \theta_{i}|\mathbf{h}|^{2 i+1}
$$

where $\delta(\mathbf{h})$ represents Kronecker delta function, $C_{0}$ and $\theta_{i}$ account for unknown coefficients, as well as $m$ indicates the number of the monomials. The model in (14) is assumed to be isotropic in this study where one can assume that the drift polynomial filter out the anisotropy [26], although many of literature have reported the influence of anisotropy in the turbulent APS [12], [14], [38]-[41]. The coefficients in (14) are constrained to $C_{0} \geq 0, \theta_{0} \leq 0, \theta_{2} \leq 0$, and $\theta_{1} \geq-\frac{10}{3} \sqrt{\theta_{0} \theta_{2}}$ in order to satisfy the conditionally positive definite of polynomial GC. Assuming that the order $k$ and the polynomial GC model in (14) are known, the problem of the GC inference downs to the determination of unknown coefficients of the GC model. The parameters $C_{0}$ and $\theta_{i}$ in (14) are iteratively estimated by the methods such that parameters are unbiased, and variance of the estimation error is minimized, namely iterative minimum variance unbiased quadratic estimation (MVUQ) algorithm [42], [43]. The further explanation of the 


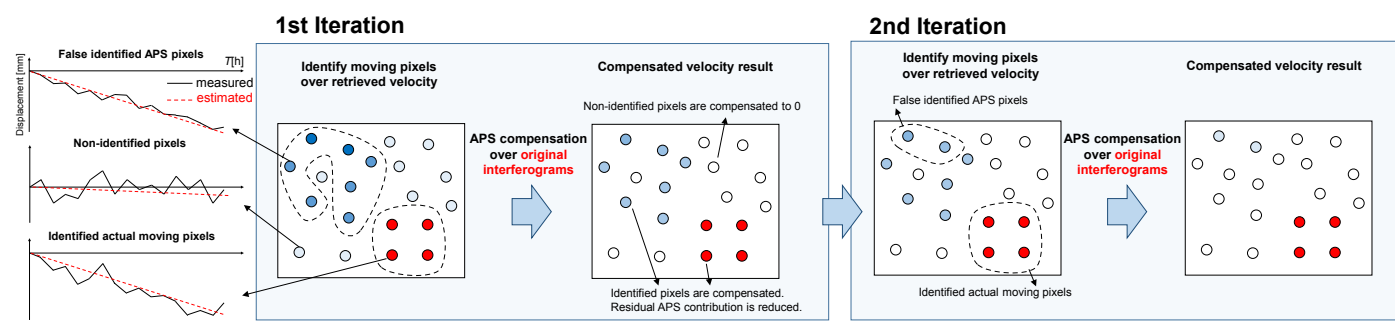

Fig. 4. A concept of the proposed iterative method. Stable pixels are shown by the circles with color strength corresponding to the velocity, where red and blue color represent the actual moving and non-moving pixels with residual APS, respectively. The time-series examples shown in the left part of the figure describe measured and estimated graphs with the black solid line and red dashed line, respectively.

GC inference is demonstrated in Appendix.

Now, we consider the spatial prediction of $\phi_{\text {res }}$ at the nonsample location $\mathbf{x}_{0}$ by a linear combination of the observed data $\phi_{\text {res }}\left(\mathbf{x}_{\alpha}\right)$,

$$
\phi_{\mathrm{res}}^{*}\left(\mathbf{x}_{0}\right)=\sum_{\alpha=1}^{n} w_{\alpha} \phi_{\mathrm{res}}\left(\mathbf{x}_{\alpha}\right)
$$

Minimizing the variance of $\left(\phi_{\text {res }}^{*}\left(\mathbf{x}_{0}\right)-\phi_{\text {res }}\left(\mathbf{x}_{0}\right)\right)$ subjects to (12), IRF- $k$ Kriging system is defined as

$$
\left\{\begin{array}{c}
\sum_{\beta}^{n} w_{\beta} K\left(\mathbf{x}_{\alpha}-\mathbf{x}_{\beta}\right)-\sum_{l=0}^{k} \mu_{l} f^{l}\left(\mathbf{x}_{\alpha}\right)=K\left(\mathbf{x}_{\alpha}-\mathbf{x}_{0}\right) \\
\sum_{\beta}^{n} w_{\beta} f^{l}\left(\mathbf{x}_{\beta}\right)=f^{l}\left(\mathbf{x}_{0}\right)
\end{array}(\alpha=1, . ., n, l=1, \ldots, L)\right.
$$

where $\mu_{l}$ represents the Lagrange multiplier. Finally, the weights can be obtained from (16) followed by inferring the $\phi_{\text {res }}^{*}\left(\mathbf{x}_{0}\right)$ by (15).

\section{An iterative DInSAR-based APS compensation}

With a priori knowledge of the moving area, APS estimation over a moving area is straightforward by extrapolating the differential phase from the known motionless pixels through the methods mentioned in Section-III A and B. Yet, moving part over the measured area is often not known. When the moving pixels are involved in the polynomial GC inference and the linear combination of the observed data to be used in the weighted average in (15) due to the false identification, a spatial prediction leads to an ill-posedness. Therefore, the remained challenge is how to identify the moving pixels and exclude them in the residual APS prediction step as well as the stratified APS estimation step.

The simple strategy is to employ a criterion of the velocity estimated by an advanced DInSAR algorithm since the estimated velocity in moving pixels is higher than non-moving pixels. Nonetheless, this is not straightforward for near-realtime GB-SAR measurements because the residual APS and possible stratified APS errors are temporally correlated which are translated to certain velocity in the final result.

A core idea of the proposed method is to iteratively find the candidates of moving pixels by an absolute velocity criterion

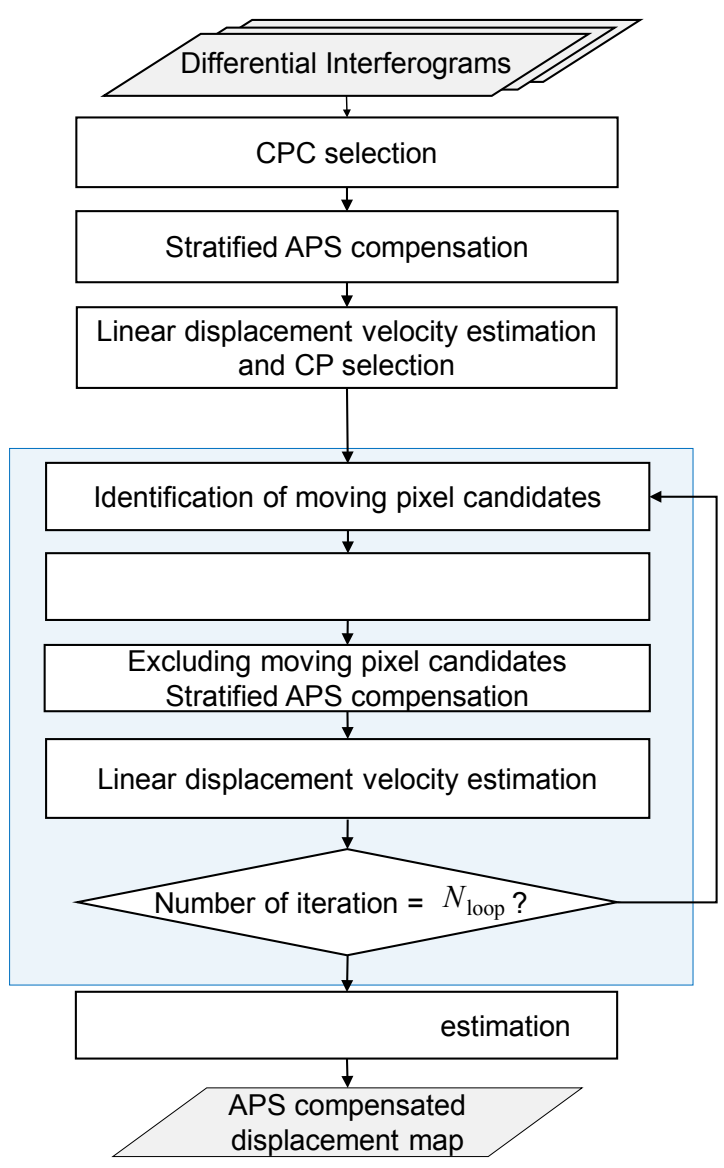

Fig. 5. The work flow of the iterative APS compensation.

based on the time-series DInSAR velocity result. Fig. 4 gives the example of the proposed iterative concept where red and blue color of pixels indicate the actual moving and non-moving pixels which have residual APS, respectively, with the color strength corresponding to the absolute velocity. In Fig. 4, we demonstrate three types of temporal behaviors; temporally correlated APS, temporally uncorrelated APS, and actual displacement which are shown in the left part of the figure. The simple velocity criterion may choose both the moving pixels and non-moving pixels which have the temporally correlated APS. The proposed method first chooses both pixels as the candidate of moving pixels (at the pixel identification of 1 st iteration in Fig. 4). Then we exclude them and compensate the residual APS in original interferograms. In order to compensate 
for the residual APS, we perform the IRF- $k$ Kriging to spatially extrapolate the APS from the non-identified pixels over the candidate of moving pixels. This process estimates the APS over the moving pixels followed by subtracting the estimated APS from each interferogram as compensation. After finishing the 1st iteration, the APS effect is suppressed over the identified pixels. Note that the accuracy of the APS estimation by Kriging prediction highly depends on the non-identified pixels. This means that if the local spatial signature of the residual APS is removed after excluding false identified pixels, Kriging prediction leads to bias prediction of a local spatial pattern of the residual APS. As a consequence, the residual APS is not adequately compensated and remained after the 1st iteration described in the compensated example of Fig. 4. The proposed method again addresses to identify the candidates of moving pixels from velocity results after the APS compensation in the 1st iteration. In the second iteration, the false identification rate is reduced thanks to the residual APS compensation in the 1st iteration as shown in the 2nd iteration of Fig. 4. Consequently, the increased number of non-identified pixels leads to better prediction of residual APS. In this way, the proposed method iteratively increases the accuracy of a moving pixel identification and prediction of APS.

The displacement retrieval section in our processing scheme is based on the Coherent Pixels Technique (CPT) developed initially been for spaceborne applications [20], [22]. The CPT also has been applied to GB-SAR applications for discontinuous (non-real-time) measurements both in urban areas [44] and distributed scatterers of natural environments [45]. In zero-baseline GB-SAR, the time-series of interferometric differential phase can be modeled by a linear displacement model $\phi_{\text {linear }}$ and $\phi_{\text {APS }}$ as well as a possible nonlinear displacement $\phi_{\text {non-linear }}$ with $M$ interferograms

$$
\begin{aligned}
\phi^{\text {measured }}\left(\mathbf{x}, T_{i}\right)= & \phi_{\text {linear }}\left(\mathbf{x}, T_{i}\right)+\phi_{\mathrm{APS}}\left(\mathbf{x}, T_{i}\right)+\phi_{\text {non-linear }}\left(\mathbf{x}, T_{i}\right) \\
= & \frac{4 \pi}{\lambda} T_{i} v(\mathbf{x})+\left(\phi_{\mathrm{str}}\left(\mathbf{x}, T_{i}\right)+\phi_{\mathrm{res}}\left(\mathbf{x}, T_{i}\right)\right) \\
& +\phi_{\text {non-linear }}\left(\mathbf{x}, T_{i}\right)
\end{aligned}
$$

where $T_{i}(i=1,2, \ldots, M)$ and $v$ account for a time-separation between slave and master images and velocity of line of sight (LOS) displacement at the 2D position of the pixel $\mathbf{x}$.

A flow chart of the proposed method is shown in Fig. 5, as well as the step by step explanations of our algorithm, are given as follows.

1) A set of the interferogram is created by available GB-SAR images acquired within a certain time span. Because the temporal decorrelation might be assumed as small in nearreal-time monitoring, a set of differential interferograms that uses the same master image is created to avoid redundant pairs which burden a computational cost.

2) The stable pixels are selected as the Coherent Pixel (CP) candidates (CPC). The mean interferometric coherence criterion along the whole interferogram stacks is adopted in our method defined as

$$
\bar{\gamma}=\frac{1}{M} \sum_{i=1}^{M}\left|\frac{\left\langle s_{1} s_{i+1}^{*}\right\rangle}{\sqrt{\left\langle s_{1} s_{i+1}^{*}\right\rangle} \sqrt{\left\langle s_{2} s_{i+1}^{*}\right\rangle}}\right|
$$

where $s_{1}$ (master image) and $s_{i+1}$ (slave image) are the complex values corresponding to the same pixel forming an interferogram, $\langle\cdot\rangle$ indicates the sample averaging with the assumptions of both the stationarity and the ergodicity which is realized by spatial averaging. The pixels with higher temporal mean coherence are selected as the CPCs.

3) The stratified APS compensation is performed by the polynomial stratified APS model in (8) using the CPC for solving the unknown coefficients. Note that the phase wrapping caused by the stratified APS along the slant range must be unwrapped before compensation. After a global APS correction with respect to a height and a slant range, the remained differential phase is expressed as

$$
\begin{aligned}
\phi_{\text {str_comp }}^{\text {measured }}\left(\mathbf{x}, T_{i}\right) & =\phi_{\text {linear }}\left(\mathbf{x}, T_{i}\right)+\phi_{\text {res }}\left(\mathbf{x}, T_{i}\right) \\
& +\phi_{\text {non-linear }}\left(\mathbf{x}, T_{i}\right)+\phi_{\text {str_error }}\left(\mathbf{x}, T_{i}\right),
\end{aligned}
$$

where $\phi_{\text {str_error }}$ is the possible stratified APS compensation error.

4) In the proposed method, the CPT is employed to estimate the linear velocity at each CPC. Following three steps are applied in the CPT for velocity estimation; (I) a connection of each CPC by Delaunay triangulation forming a spatial network, (II) an estimation of the velocity in each connected arc towards minimizing a residual APS effect, (III) an integration of the estimated velocity in each arc to compute the absolute velocity at CPCs. The explicit explanation of the velocity estimation can be referred to [22]. At (II), the temporal coherence is evaluated in each arc. The arcs indicating lower temporal coherence than the pre-defined threshold are removed and only remained arcs are integrated at (III). Consequently, the remained pixels after (III) are called CPs.

5) The CPs with an absolute velocity higher than the determined threshold $T h_{\text {velocity }}$ are identified as candidates of moving pixels. After identification, we exclude the identified pixels from all interferograms.

6) The polynomial GC is inferred using non-identified CPs. Subsequently, we predict the residual APS by performing the IRF- $k$ Kriging interpolation over the identified pixels. These processes are applied to all interferograms. After a prediction of the residual APS, we subtract them from the original data to compensate residual APS as,

$$
\begin{aligned}
\phi_{\text {res_comp }}^{\text {measured }}\left(\mathbf{x}, T_{i}\right)= & \phi^{\text {measured }}\left(\mathbf{x}, T_{i}\right)-\phi_{\text {res }}\left(\mathbf{x}, T_{i}\right) \\
= & \phi_{\text {linear }}\left(\mathbf{x}, T_{i}\right)+\phi_{\text {str }}\left(\mathbf{x}, T_{i}\right)+\phi_{\text {non-linear }}\left(\mathbf{x}, T_{i}\right) \\
& +\phi_{\text {res_error }}\left(\mathbf{x}, T_{i}\right),
\end{aligned}
$$

where $\phi_{\text {res error }}$ is the residual APS compensation error.

Note that only $\phi_{\text {str }}$ is presented as the APS in (20).

7) We then compensate $\phi_{\text {str }}$ by the polynomial stratified APS model. In the estimation step of the coefficients $\beta$, 


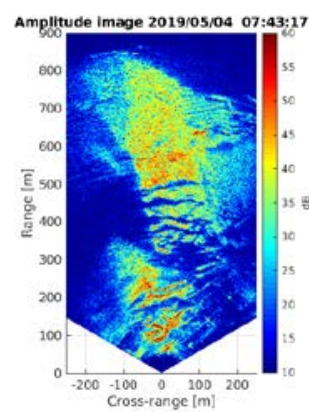

(a)

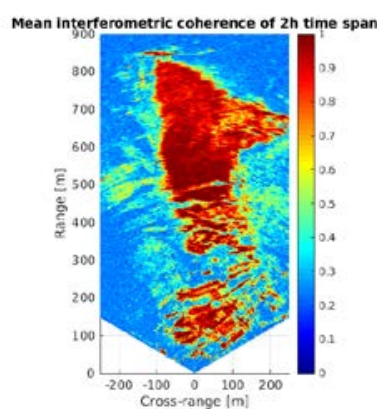

(b)
Fig. 6. (a) GB-SAR HH polarization reflectivity image of the landslide affected mountainous slope located in the Aso-mountain acquired at 7:43, 4th May, 2019; (b) Mean interferometric coherence processed by 25 images with $5 \mathrm{~min}$ time interval acquired within two-hours from 5:48 to 7:48.

the moving pixels are excluded from the interferograms in (20) so that we can avoid the undesired effect of displacement signals for solving the stratified APS model. After the compensation, the interferograms are expressed as

$$
\begin{aligned}
& \phi_{\text {res_str_comp }}^{\text {measured }}\left(\mathbf{x}, T_{i}\right) \\
& =\phi_{\text {linear }}\left(\mathbf{x}, T_{i}\right)+\phi_{\text {non-linear }}\left(\mathbf{x}, T_{i}\right)+\phi_{\text {res_str_error }}\left(\mathbf{x}, T_{i}\right),
\end{aligned}
$$

where $\phi_{\text {res_str_error }}$ includes the error of both the residual and the stratified APS compensation.

8) The phase of the linear displacement $\phi_{\text {linear est }}$ is again estimated by CPT technique.

9) Repeat the steps from 5) to 8) until the specified number of loops $N_{\text {loop }}$.

10) After finishing an iteration, the phase residue is calculated by subtracting the estimated linear model $\phi_{\text {linear_est }}$ from the interferometric phase as

$$
\begin{aligned}
\phi_{\text {residue }}\left(\mathbf{x}, T_{i}\right) & =\phi_{\text {res_str_comp }}^{\text {measured }}\left(\mathbf{x}, T_{i}\right)-\phi_{\text {linear_est }}\left(\mathbf{x}, T_{i}\right) \\
& =\phi_{\text {non_linear }}\left(\mathbf{x}, T_{i}\right)+\phi_{\text {res_str_error }}\left(\mathbf{x}, T_{i}\right),
\end{aligned}
$$

Since APS has already been compensated, the phase residue in (22) represents the non-linear component. The final product includes both linear and non-linear displacement.

\section{EXPERIMENTAL RESULTS}

In this paper, the APS compensation is developed mainly for the near-real-time measurement scheme in the framework of continuous operation. Notably, we consider the fast displacement possibly occurred within a short period. Thus it is desirable to validate the performance of the proposed method by means of a series of GB-SAR images acquired within a short period. First, the performance of the proposed method through the simulated displacement with presented APS over the test area is presented. For this purpose, we use two hours of data acquired from 05:48 to 07:48 on 4th May 2019 because these data include severe APS fluctuation. During this time-span, our GB-SAR obtains 25 images in total with a $5 \mathrm{~min}$ time interval. Afterward, the dataset with soil erosion over the earthwork area

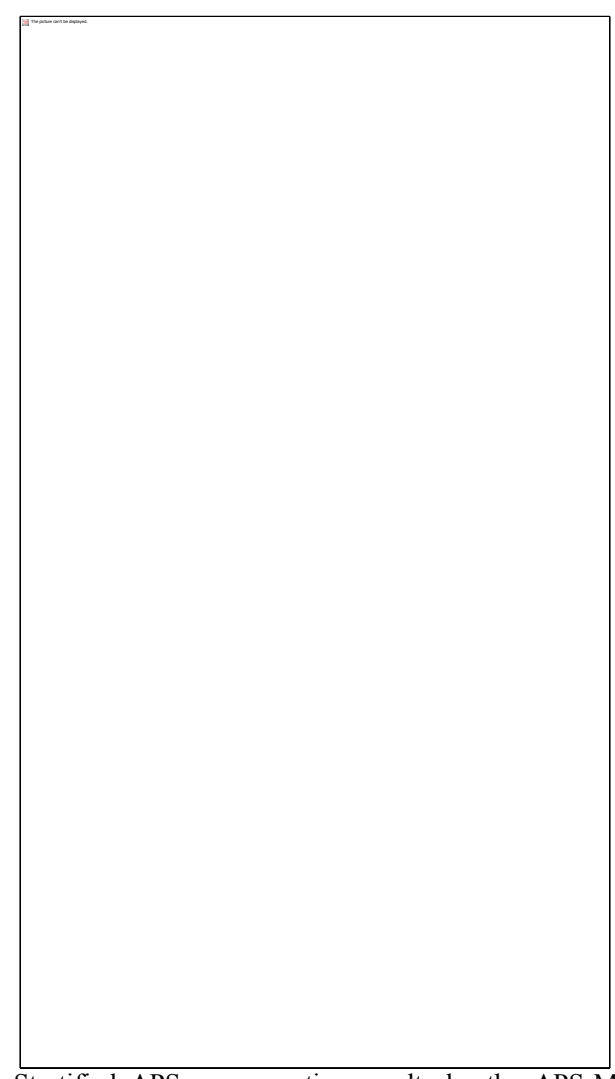

Fig. 7. Stratified APS compensation results by the APS-MRM for interferometric pair1 ((a) and (c)) and pair2((b) and (d)). (a), (b) (black dot) Differential phase and (red circle) estimated stratified APS projected on the range axis.

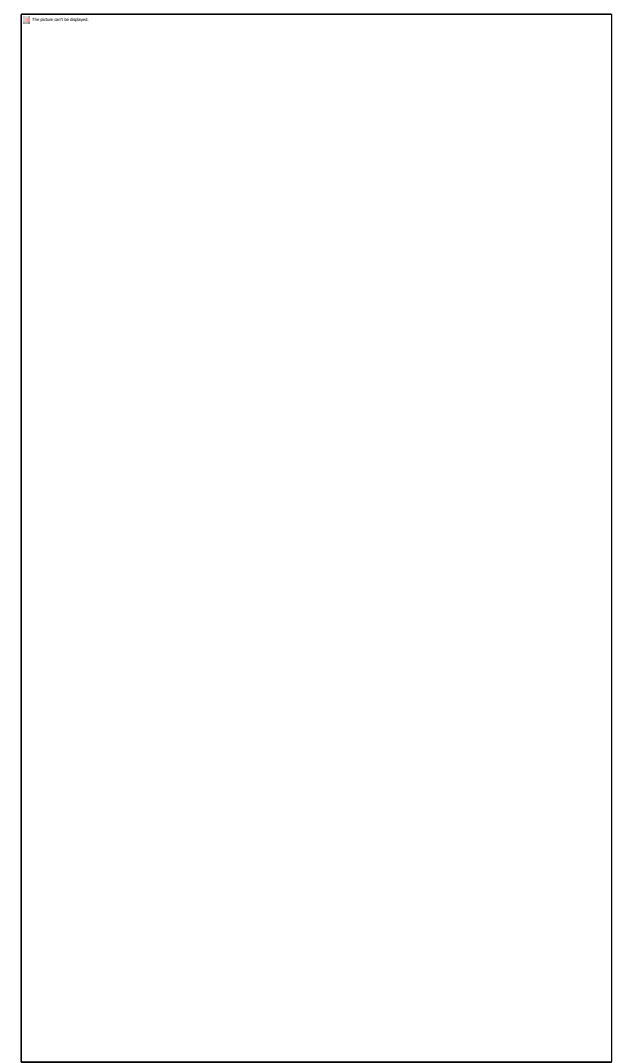

Fig. 8. Stratified APS compensation results by the proposed polynomial model for interferometric pairl ((a) and (c)) and pair2((b) and (d)). 


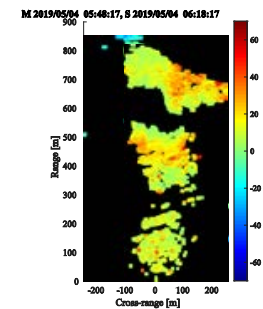

(a)

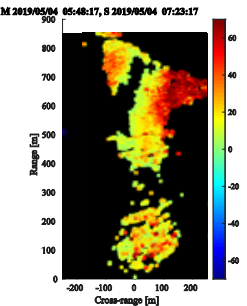

(b)

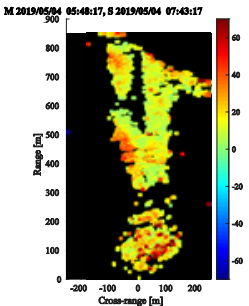

(c)
Fig. 9. (a)-(c) Examples of the residual APS after applying the stratified APS compensation. The labels on the top of each figure indicate the acquisition time of the master and slave GB-SAR images with the format year-month-day-hour-minute.

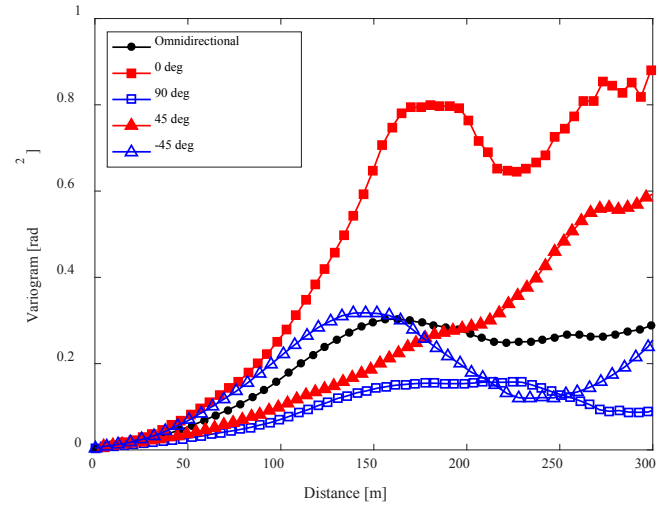

Fig. 10. Derived experimental variogram for different angles from the interferometric pair of Fig. 9(b) where direction of 0deg corresponds to cross-range direction.

is used for evaluation. Thus three types of datasets in total are presented for validation purposes.

In Fig. 6(a) and (b), we show the GB-SAR reflectivity image of HH-polarization and the mean interferometric coherence processed by 25 images from $05: 48$ to $07: 48$ on 4th May 2019 as a supplement to visually understand the radar image and temporal stability in the test area.

\section{A. Investigation of the stratified APS}

The performance of the polynomial stratified APS model is first evaluated. In this evaluation, the pixels with mean interferometric coherence higher than 0.85 from the $5 \times 3$ (range $\times$ cross-range) multi-looked images, are used for solving the unknown coefficients $\beta$ of the polynomial model in (8). The two pairs of the measured differential phase are selected for visualization of estimated phase change of both the APS-MRM and the polynomial stratified APS model along with a slant range. The first interferometric pair (pair1) is formed by images acquired at 05:48 and 07:03, 29th Nov. 2019. While the second interferometric pair (pair2) is formed by images acquired at 05:48 and 07:13, 29th Nov. 2019 (pair2).

First, we present the estimation and compensation results by the APS-MRM model. Fig. 7(a) and (b) show the differential phase changes and the estimated APS by the APS-MRM projected on a range axis for the two interferometric pairs. Notice how both global differential phase trends in Fig. 7(a) and (b) are not estimated by the APS-MRM where the measured phase difference and the estimated APS show a significant error between them. This error implies that a linear refractivity distribution model in (5) is no longer valid in our area. As a result, the phase residues of the stratified APS remain in the compensated GB-SAR images shown in Fig. 7(c) and (d).

On the other hand, Fig. 8 shows the differential phase changes and the estimated APS by the polynomial stratified APS model in (8) projected on a range axis for the pair1 and pair2. Global trends of the stratified APS for both pairs are now compensated. Notice that the stratified APS errors remained in Fig. 8 (c) and (d) are much smaller than the errors in Fig. 7 (c) and (d), yielding an improvement of the presented stratified APS modeling.

\section{B. The residual APS images and those spatial variogram}

Three examples of the interferograms after compensating the stratified APS taken on 4th May are shown in Fig. 9(a)-(c), respectively. Since a global trend with respect to a slant range and a topographic height are removed, a lateral variation and a local change of the refractivity are presented. Local blob shapes of the residual APS are found in Fig. 9(a) and (b) while a slow variation is exhibited in Fig. 9(b). Such the slow variation behaves as a drift component in the RF. To illustrate the stationarity of the residual APS in Fig. 9(b), the omnidirectional and directional variogram of $0 \mathrm{deg}$ (parallel to cross-range direction), 45deg, -45deg, and 90deg are shown in Fig. 10. Notice that the directional-variogram shows a strong variation in terms of the direction. Especially, directional-variograms in 0 deg and $45 \mathrm{deg}$ show a hyperbolic curve, which implies a presence of the drift component, representing the space-varying mean of the RF [26]. This result states that our data exhibit the non-stationarity of the RF.

\section{Evaluation through the simulated displacement}

In order to compare between retrieved displacement velocity with true value, we simulate the linear displacement with the maximum velocity of $-1 \mathrm{~mm} / \mathrm{h}$ where the negative sign corresponds to the direction from the target to the GB-SAR sensor, as well as a spatial extent of the simulated displacement is expressed by a 2D Gaussian function.

In this evaluation, the threshold of mean interferometric coherence is chosen to be 0.85 from the $5 \times 3$ (range $\times$ crossrange) multi-looked images for $\mathrm{CPC}$ selection. As a result, 82020 CPs are finally chosen based on the temporal coherence criterion whose threshold is 0.96 . In addition, the proposed method needs to determine a threshold of the absolute velocity $T h_{\text {velocity }}$ at the moving pixel candidate identification step. In fact, the accuracy of the compensated results depends on $T h_{\text {velocity }}$ as discussed in the after-mentioned Section V. In this section, we select $0.1 \mathrm{~mm} / \mathrm{h}$ as the $T h_{\text {velocity }}$. Besides, the selection of neighbor pixels to be included in the estimation by the IRF- $k$ Kriging is an important issue. In theory, the minimum mean square error is achieved when all pixels are included [26], but this is not practical from the processing-time point of view. Hence in this evaluation, 300 non-identified CPs are randomly selected within the range of $200 \mathrm{~m}$ at each estimated pixel.

Fig. 11 (a)-(c) show the APS compensated velocity at CPs 

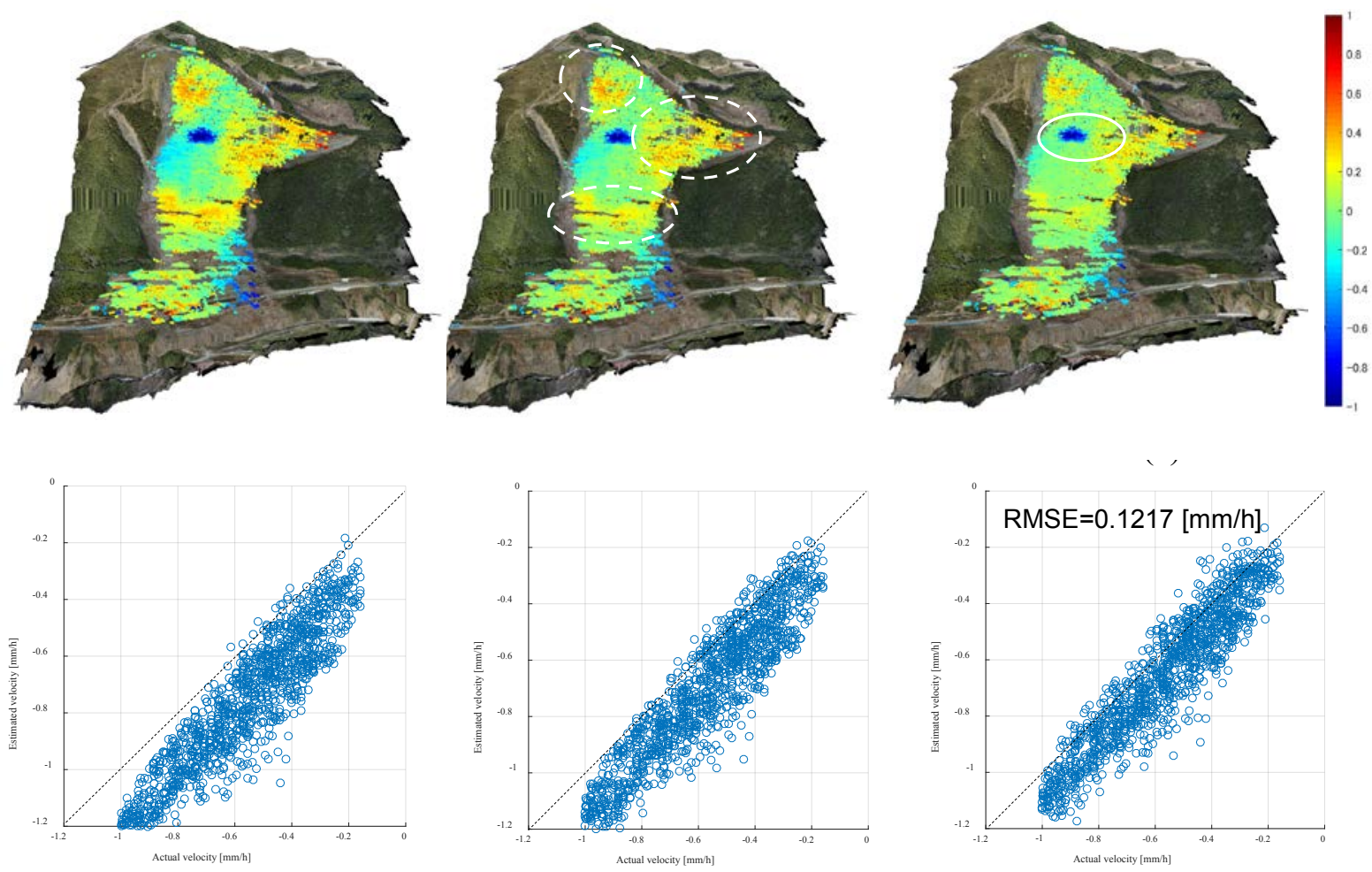

(f)

Fig. 11. (a)-(c) APS compensated velocity results at CPs by both the CPT and the proposed method projected on the DEM; and (d)-(f) scatter plots of the actual velocity versus the estimated velocity in the simulated displacement pixels. (a) and (d) Results obtained by the CPT; (b) and (e) the proposed method at the first iteration; (c) and (f) the proposed method at the second iteration. White dashed circle in (b) indicates the residual error in the first iteration, and the white solid circle indicates the simulated displacement location.

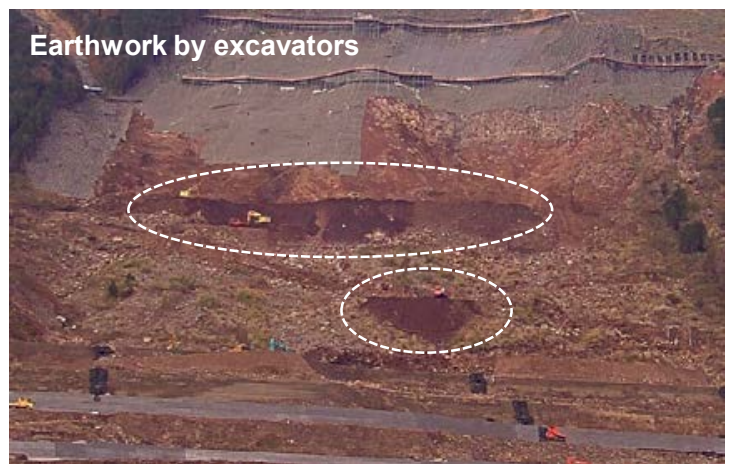

Fig. 12. The areas introducing differential phase after the earthwork due to the wind-driven soil erosion indicated by white dash circles.

projected on DEM. The estimated velocity result before a residual APS compensation is shown in Fig. 11 (a) where this is equivalent to the linear velocity of the CPT method for GBSAR described in [45] (hereafter we simply denote as CPT). While the compensation results by the proposed method at the first and the second iteration are shown in Fig. 11(b) and (c), respectively. Note that the simulated displacement is added to the middle of the slope indicated by a solid circle in Fig. 11(c). In addition, root-mean-square (RMS) in non-moving pixels is described in Fig. 11(a)-(c) as an APS error index. From Fig. 11(a), we notice that the residual errors are presented in the whole slope area. This result clearly reveals that the residual
APS in each interferogram is translated to errors in a linear velocity estimation in the CPT showing RMS of $0.2 \mathrm{~mm} / \mathrm{h}$. While the results after compensation of the residual APS by the proposed method in Fig. 11(b) and (c) yield less RMS than Fig. 11(a). Although some velocity errors can be found in the first iteration indicated by the dashed white circle in Fig. 11(b), these errors are successively suppressed in the second iteration.

In order to validate the accuracy of the velocity estimation, the scatter plot for Fig. 11(a), (b), and (c) between the actual simulated velocity and the estimated velocity are exhibited in Fig. 11(d), (e), and (f), respectively. Notice that the CPT method results in a bias estimation of the displacement velocity due to the presented residual APS. On the other hand, the scatter plots for the first and the second iteration of the proposed method show a smaller bias than the CPT. As a quantitative analysis, RMS error (RMSE) is derived for all results in Fig. 11. The proposed method of first and the second iteration yield RMSE of $0.10 \mathrm{~mm} / \mathrm{h}$ and $0.07 \mathrm{~mm} / \mathrm{h}$, while the CPT shows $0.23 \mathrm{~mm} / \mathrm{h}$. This comparison analysis demonstrates that the proposed method improves the APS compensation.

\section{Evaluation through the dataset with soil erosion}

The GB-SAR operated by Tohoku University has been employed to monitor the displacement over the landslide affected mountainous slope as an early warning issue. We have conducted this campaign for operators who work on earthwork. They routinely use the excavators as the earthwork. As a part of 


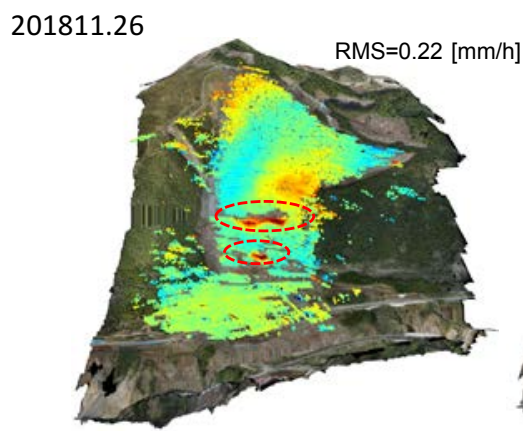

(a)

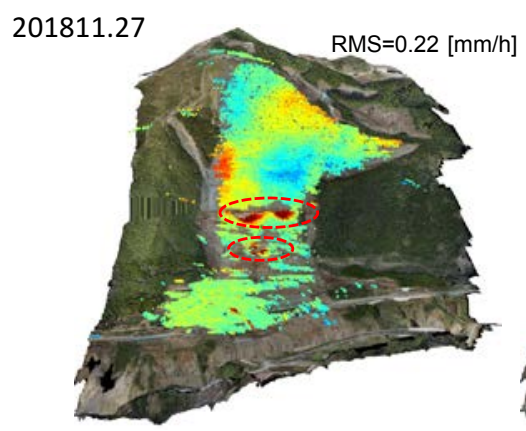

(d)

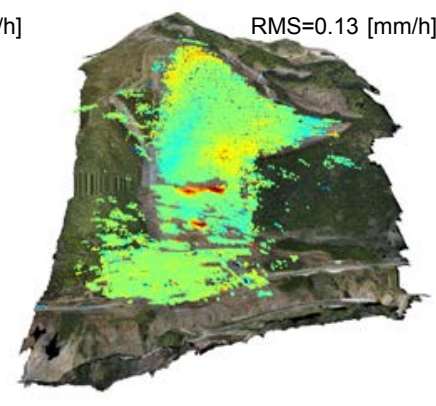

(b)

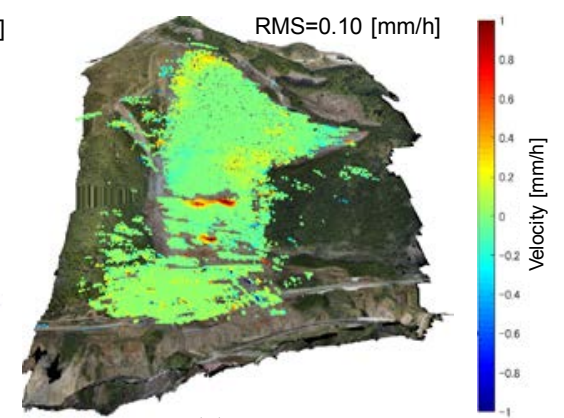

(c)

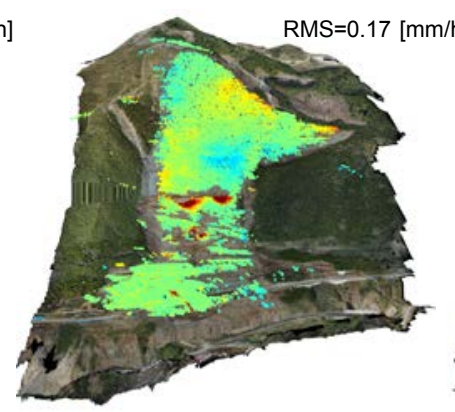

(e)

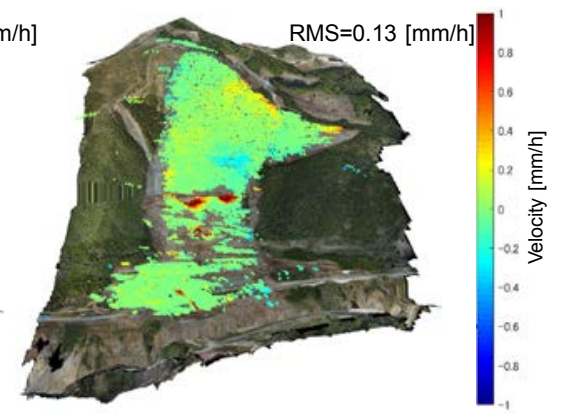

(f)

Fig. 13. The retrieved velocity results with soil erosion indicated by red circles in (a) and (d) at CPs by both the CPT and the proposed method projected on the DEM obtained by datasets on (a)-(c) 26th and (d)-(f) 27th November, 2018. (a) and (d) Results obtained by the CPT; (b) and (e) the proposed method at the first iteration; (c) and (f) the proposed method at the second iteration. RMS of APS affected pixels are shown in each figure.
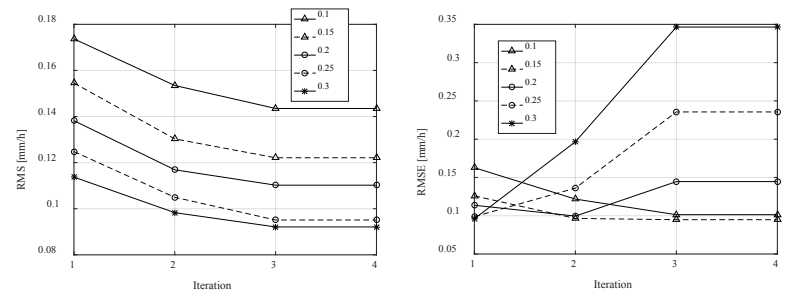

Fig. 14. The error values as changing velocity threshold. (a) The RMS values at non-moving pixels; (b) RMSE values at moving pixels.

this work, several excavators constructed the paths on the slope by filling soil. In fact, the phase difference is daily measured over soil accumulated location after finishing earthwork, which might be caused by wind-driven soil erosion. Fig. 12 shows the earthwork location indicating phase difference in the test area. We herein use datasets which include the differential phase of soil erosion for validation purposes. Among the dataset with soil erosion, we choose the two datasets measured at 17:58 to 19:58 on 26th as well as 15:58 to 17:58 on 27th Nov. 2019 because those show significant heterogeneous APS. The retrieved velocity results of those datasets are shown in Fig. 13 (a)-(c) and Fig. (d)-(f) respectively with the velocity threshold $T h_{\text {velocity }}$ of $0.1 \mathrm{~mm} / \mathrm{h}$ where Fig. 13 (a) and (d) represents results by the CPT, (b) and (e) are the results by the proposed method at the first iteration as well as (c) and (f) show second iteration of the proposed method for datasets of 26th and 27th. Note that the red circles in Fig. 13(a) and (d) indicate soil erosion locations, corresponding to the white circle in Fig. 12. In addition, we derive the RMS over the pixels except for earthwork locations for a quantitative comparison of APS error, described in each result. All the results in Fig. 13 clearly show a certain velocity in those circled earthwork locations. Nonetheless, the CPT results in Fig. 13(a) and (d) include the APS effect over the whole slope area with the RMS of $0.22 \mathrm{~mm} / \mathrm{h}$ and $0.22 \mathrm{~mm} / \mathrm{h}$, respectively. While the proposed compensation reduces the APS effect which can visually be understood from Fig. 13 (b), (c), (e), and (f) as well as suppressed RMS. For both datasets, the results by the proposed method with the second iteration yield lower RMS than those with the first iteration. From this fact based on the experimental results, it is clear that the iteration strategy within our scheme leads to the improvement of the final velocity result.

\section{DISCUSSION}

The above evaluation reveals the reasonable compensation results of the proposed method with fixed threshold values which are the key factor to determine the final performance. Here we discuss the effectiveness of the velocity threshold value in the final velocity estimation. Furthermore, the feasibility of implementation into a near-real-time monitoring scheme is noted. 


\section{A. Velocity threshold effect}

In order to visualize the dependency of the velocity threshold in our method, we quantitatively evaluate the RMS in nonmoving pixels as well as RMSE in moving pixels, shown in Fig. 14(a) and (b), respectively where we evaluate through the dataset mentioned in Section-IV C with simulated displacement. In this evaluation, we test the proposed method until 4-times the iteration. In Fig. 14(a), RMS of non-moving pixels shows a clear trend of error suppression as increasing the number of iteration. Besides, the RMS becomes lower when we choose a higher velocity threshold value. However, the RMSE of moving pixels reveals the increasing trend of error for the threshold of 0.25 and 0.3. In addition, the RMSE for the threshold of 0.2 shows an error increase from the third iteration. This error propagation might be caused because some of the moving pixels are involved in the Kriging system. In this case, the Kriging results in the bias estimate, degrading the compensation accuracy. Hence, the trade-off exists in our method between RMS in non-moving pixels and RMSE in moving-pixels, and the velocity threshold needs to be carefully considered.

\section{B. Feasibility of near-real-time measurement}

We present the fully automatic APS compensation algorithm which is necessary for a continuous operational mode. Since our method based on CPT, a certain number of GB-SAR images are required. Therefore, a near-real-time concept, in this case, means estimating the APS compensated displacement with the shortest delay possible after stacking GB-SAR images [25], which is an important consideration for an early warning issue. The system performs the proposed method when $M$ images are stacked and wait until the next $M$ images. Time-series displacement is thus obtained by accumulating the displacement estimated in each group of the image stack. Hence, in principle, the more increase acquisition, the shorter delay of each processing can be realized. In practice, a computational cost in each compensation has to be dealt with where it must not exceed the period between each processing. A computational cost of our proposed method highly depends on several factors such as the number of interferograms, the number of CPC, the number of iteration $N_{\text {loop }}$, as well as the number of neighbor pixels $n$ at the estimation step of the $\phi_{\text {res }}^{*}\left(\mathbf{x}_{0}\right)$ in (15). Besides the velocity threshold, we need to deal with the above factors which consequently improve the total system performance in a near real-time monitoring scheme.

\section{CONCLUSION}

A novel APS compensation for a near-real-time GB-SAR measurement over a mountainous area has been proposed in this paper.

The higher variation of meteorological conditions is revealed by in-situ meteorological measurements due to the basin structure of the target area formed by the outer rim of the caldera and the volcano cone.

As for the stratified APS, the conventional APS-MRM technique is modified accordingly for the case with the presence of a high spatial inhomogeneous refractivity by introducing the 2D quadratic polynomial function of the refractivity index with respect to a slant range and a topographic height. With the polynomial GC model, the residual APS components including longer scale components and short-scale are predicted by the IRF- $k$ Kriging interpolation, taking into account the nonstationarity. In the framework of the proposed method, the candidates of moving pixels are iteratively identified by an absolute velocity criterion estimated by the CPT.

The proposed method has been tested with GB-SAR data acquired over the test area. In this evaluation, the proposed polynomial stratified APS model demonstrated the reasonable estimation and compensation capability of a global differential phase trend. The proposed APS compensation scheme yielded lower APS error and higher estimation accuracy of the simulated displacement than the CPT. The analysis through the datasets with soil erosion over the earthwork shows APS error suppression with the proposed method. Furthermore, the results demonstrate the applicability of an iterative strategy of our method where the results are improved with increasing iteration. However, improper setting of the velocity threshold reveals the degradation of results with an increase of the iteration. Therefore, the velocity threshold should be carefully determined based on the knowledge of the predicted displacement phenomenon.

Finally, the practical consideration in terms of the computational cost of the proposed compensation processing is noted. This is important to realize the near-real-time monitoring scheme which is highly required for the early-warning issue.

\section{APPENDIX}

\section{Practical Determination of Generalized Covariance}

Prior to an adaptation of the IRF- $k$ Kriging, several steps are required to determine the order $k$ and the GC model in practice. First, we need to perform the trend analysis so that the order $k$ of the trend can be determined. In this process, we iteratively find the optimal order $k$ of the polynomial by evaluating the residual phase after subtracting the fitted order $k$ polynomial from the original phase. We then define the optimal structure of the GC. Among all possible GC structures, following five models of polynomial GC are practically evaluated;

$$
\left\{\begin{array}{l}
K(\mathbf{h})=C_{0} \delta(\mathbf{h}) \\
K(\mathbf{h})=\theta_{0}|\mathbf{h}| \\
K(\mathbf{h})=C_{0} \delta(\mathbf{h})+\theta_{0}|\mathbf{h}| \\
K(\mathbf{h})=\theta_{0}|\mathbf{h}|+\theta_{1}|\mathbf{h}|^{3} \\
K(\mathbf{h})=\theta_{0}|\mathbf{h}|+\theta_{1}|\mathbf{h}|^{3}+\theta|\mathbf{h}|^{5}
\end{array}\right.
$$

We further perform cross-validation to choose the optimal GC model. In cross-validation, APS are predicted at test data pixels chosen within observed data sets, and errors between the predicted and the measured values are calculated, referred to the mean squared error of prediction (MSEP) as

$$
\mathrm{MSEP}=\frac{1}{m} \sum_{i=1}^{m}\left[\phi_{\mathrm{res}}^{*}\left(\mathbf{x}_{i}\right)-\phi_{\mathrm{res}}\left(\mathbf{x}_{i}\right)\right]^{2}
$$

Subsequently, the model minimizing the MSEP is chosen as 
the optimal GC model. After all the above procedures, the IRF$k$ Kriging is finally applied to estimate the $\phi_{\text {res }}$.

\section{ACKNOWLEDGMENT}

This work was supported by JSPS Grand-in-Aid for Scientific Research (A) 23246076 and Grand-in-Aid for JSPS Research Fellows 18J20104. We also thank Ministry of land, infrastructure, Transport and Tourisum Kyushu Regional Development Bureau and Kumagai Gumi for their support in field site and providing us local information. We also would like to thank Prof. Yamazaki of the Tohoku University for fruitful discussion about the mesoscale weather condition. In addition, the authors thank Dr. Giovanni Nico for his advice about the APS compensation method.

\section{REFERENCES}

O. Monserrat, M. Crosetto, and G. Luzi, "A review of ground-based SAR interferometry for deformation measurement," ISPRS $J$. Photogramm. Remote Sens., vol. 93, pp. 40-48, 2014.

[2] R. Caduff, F. Schlunegger, A. Kos, and A. Wiesmann, "A review of terrestrial radar interferometry for measuring surface change in the geosciences," Earth Surf. Process. Landforms, vol. 40, no. 2, pp. 208-228, 2015.

[3] G. Luzi et al., "Ground-based radar interferometry for landslides monitoring: Atmospheric and instrumental decorrelation sources on experimental data," IEEE Trans. Geosci. Remote Sens., vol. 42, no. 11, pp. 2454-2466, 2004.

[4] L. Pipia, X. Fàbregas, A. Aguasca, and C. López-Martínez, "Atmospheric artifact compensation in ground-based DInSAR applications," IEEE Geosci. Remote Sens. Lett., vol. 5, no. 1, pp. 88-92, 2008.

[5] L. Noferini et al., "Permanent scatterers analysis for atmospheric correction in ground-based SAR interferometry," IEEE Trans. Geosci. Remote Sens., vol. 43, no. 7, pp. 1459-1470, 2005.

[6] R. Iglesias et al., "Atmospheric Phase Screen Compensation in Ground-Based SAR With a Multiple-Regression Model Over Mountainous Regions," IEEE Trans. Geosci. Remote Sens., vol. 52, no. 5, pp. 2436-2449, 2014.

[7] L. Iannini and A. Monti Guarnieri, "Atmospheric phase screen in ground-based radar: Statistics and compensation," IEEE Geosci. Remote Sens. Lett., vol. 8, no. 3, pp. 537-541, 2011.

[8] S. Rödelsperger, A. Coccia, D. Vicente, and A. Meta, "Introduction to the new metasensing ground-based SAR: Technical description and data analysis," in 2012 IEEE International Geoscience and Remote Sensing Symposium, 2012, pp. 4790-4792.

[9] N. Dematteis, G. Luzi, D. Giordan, F. Zucca, and P. Allasia, "Monitoring Alpine glacier surface deformations with GB-SAR," Remote Sens. Lett., vol. 8, no. 10, pp. 947-956, 2017.

[10] C. Atzeni, M. Barla, M. Pieraccini, and F. Antolini, "Early Warning Monitoring of Natural and Engineered Slopes with Ground-Based Synthetic-Aperture Radar," Rock Mech. Rock Eng., vol. 48, no. 1, pp. 235-246, 2015.

[11] R. Caduff, A. Kos, F. Schlunegger, B. W. McArdell, and A. Wiesmann, "Terrestrial radar interferometric measurement of hillslope deformation and atmospheric disturbances in the Illgraben debris-flow catchment, Switzerland," IEEE Geosci. Remote Sens. Lett., vol. 11, no. 2, pp. 434-438, 2014.

[12] Z. Li et al., "Time-series InSAR ground deformation monitoring: Atmospheric delay modeling and estimating," Earth-Science Rev., vol. 192, no. December 2018, pp. 258-284, 2019.

[13] R. F. Hanssen, "Radar Interferometry: Data interpretation and error analysis," Ph.D. dissertation, Delft University of Technology, The Netherlands, 2001.

[14] Z. W. Li, X. L. Ding, C. Huang, Z. R. Zou, and Y. L. Chen, "Atmospheric effects on repeat-pass InSAR measurements over Shanghai region," J. Atmos. Solar-Terrestrial Phys., vol. 69, no. 12, pp. 1344-1356, 2007.
[15] S. Baffelli, "Development of Calibration Methods For A Ku-Band Polarimetric Terrestrial Radar Interferometer," Ph. D. dissertation, Department of Civil, Environmental and Geomatic Engineering, ETH Zürich, Zürich, Switzerland, 2019.

[16] G. Nico, R. Tome, J. Catalao, and P. M. A. Miranda, "On the use of the WRF model to mitigate tropospheric phase delay effects in SAR interferograms," IEEE Trans. Geosci. Remote Sens., vol. 49, no. 12, pp. 4970-4976, Dec. 2011.

[17] P. Mateus, G. Nico, and J. Catalao, "Uncertainty Assessment of the Estimated Atmospheric Delay Obtained by a Numerical Weather Model (NMW)," IEEE Trans. Geosci. Remote Sens., vol. 53, no. 12, pp. 6710-6717, Dec. 2015.

[18] A. Ferretti, C. Prati, F. Rocca, and I. Politecnico, "Permanent scatterers in SAR interferometry," IEEE Trans. Geosci. Remote Sens., vol. 39, no. 1, pp. 1528-1530, 1999.

[19] A. Ferretti, C. Prati, and F. Rocca, "Nonlinear subsidence rate estimation using permanent scatterers in differential SAR interferometry," IEEE Trans. Geosci. Remote Sens., vol. 38, no. 5, pp. 2202-2212, 2000.

[20] O. Mora, J. J. Mallorqui, and A. Broquetas, "Linear and nonlinear terrain deformation maps from a reduced set of interferometric SAR images," IEEE Trans. Geosci. Remote Sens., vol. 41, no. 10 PART I, pp. 2243-2253, 2003.

[21] P. Berardino, G. Fornaro, R. Lanari, and E. Sansosti, "A new algorithm for surface deformation monitoring based on small baseline differential SAR interferograms," IEEE Trans. Geosci. Remote Sens., vol. 40, no. 11, pp. 2375-2383, 2002.

[22] P. Blanco-Sánchez, J. J. Mallorquí, S. Duque, and D. Monells, "The coherent pixels technique (CPT): An advanced DInSAR technique for nonlinear deformation monitoring," Pure Appl. Geophys., vol. 165, no. 6, pp. 1167-1193, 2008.

[23] J. Dong, L. Zhang, M. Liao, and J. Gong, "Improved correction of seasonal tropospheric delay in InSAR observations for landslide deformation monitoring," Remote Sens. Environ., vol. 233, no. August, pp. 1-18, 2019.

[24] S. Rödelsperger, "Real-time Processing of Ground Based Synthetic Aperture Radar (GB-SAR) Measurements," Ph.D. dissertation, Institut für Physikalische Geodäsie, Technische Universität Darmstadt, Darmstadt, Germany, 2011.

[25] C. Hu, Y. Deng, W. Tian, and J. Wang, "A PS processing framework for long-term and real- time GB-SAR monitoring," Int. J. Remote Sens., vol. 40, no. 16, pp. 6298-6314, 2019.

[26] J.-P. Chiles, Geostatistics : modeling spatial uncertainty, 2nd ed. Wiley, 2011.

[27] V. I. Tatarski, Wave Propagation in a Turbulent Medium. New York: USA:McGraw-Hill, 1961.

[28] J. Butt, A. Wieser, and S. Conzett, "Intrinsic random functions for mitigation of atmospheric effects in terrestrial radar interferometry," J. Appl. Geod., vol. 11, no. 2, pp. 89-98, 2017.

[29] H. Wackernagel, Multivariate Geostatistics. Berlin, Heidelberg: Springer Berlin Heidelberg, 2003.

[30] E. K. Smith and S. Weintraub, "The Constants in the Equation for Atmospheric Refractive Index at Radio Frequencies," Proc. IRE, vol. 41, no. 8, pp. 1035-1037, 1953.

[31] J. Kondo, T. Kuwagata, and S. Haginoya, "Heat Budget Analysis of Nocturnal Cooling and Daytime Heating in a Basin," Journal of the Atmospheric Sciences, vol. 46, no. 19. pp. 2917-2933, 2002.

[32] Y. Kurose, K. Ohba, A. Maruyama, and T. Maki, "Characteristics of Local Wind 'Aso Oroshi'.," J. Agric. Meteorol., vol. 58, no. 2, pp. 93-101, 2002.

[33] J. Schmidli and R. Rotunno, "Mechanisms of Along-Valley Winds and Heat Exchange over Mountainous Terrain," J. Atmos. Sci., vol. 67, no. 9, pp. 3033-3047, 2010.

[34] C. D. Whiteman et al., "Inversion Breakup in Small Rocky Mountain and Alpine Basins," J. Appl. Meteorol., vol. 43, no. 8, pp. 1069-1082, 2004.

[35] ITU, "The radio refractive index: its formula and refractivity data," 2015.

[36] G. Matheron, "THE INTRINSIC RANDOM FUNCTIONS AND THEIR APPLICATIONS," Appl. Probab., vol. 5, no. 3, pp. 439468, 1973.

[37] P. Delfiner, "Linear Estimation of non Stationary Spatial Phenomena," in Advanced Geostatistics in the Mining Industry, 1976, pp. 49-68. 
[38] M. Yaseen, N. A. S. Hamm, V. Tolpekin, and A. Stein, "Anisotropic kriging to derive missing coseismic displacement values obtained from synthetic aperture radar images," J. Appl. Remote Sens., vol. 7, no. 1, pp. 1-18, 2013.

[39] J. Wei, Z. Li, J. Hu, G. Feng, and M. Duan, “Anisotropy of atmospheric delay in InSAR and its effect on InSAR atmospheric correction," J. Geod., vol. 93, no. 2, pp. 241-265, 2019.

[40] S. H. G. Knospe and S. Jónsson, "Covariance estimation for dInSAR surface deformation measurements in the presence of anisotropic atmospheric noise," IEEE Trans. Geosci. Remote Sens., vol. 48, no. 4, pp. 2057-2065, 2010.

[41] A. Refice, A. Belmonte, F. Bovenga, and G. Pasquariello, "On the use of anisotropic covariance models in estimating atmospheric DInSAR contributions," IEEE Geosci. Remote Sens. Lett., vol. 8, no. 2, pp. 341-345, 2011.

[42] P. K. Kitanidis, "Statistical estimation of polynomial generalized covariance functions and hydrologic applications," Water Resour. Res., vol. 19, no. 4, pp. 909-921, 1983.

[43] P. K. Kitanidis, "Minimum-variance unbiased quadratic estimation of covariances of regionalized variables," J. Int. Assoc. Math. Geol., vol. 17 , no. 2, pp. 195-208, 1985.

[44] L. Pipia et al., "Polarimetric Differential SAR Interferometry : First Results With Ground-Based Measurements," IEEE Geosci. Remote Sens. Lett., vol. 6, no. 1, pp. 167-171, 2009.

[45] R. Iglesias et al., "Ground-Based Polarimetric SAR Interferometry for the Monitoring of Terrain Displacement Phenomena - Part I: Theoretical Description," IEEE J. Sel. Top. Appl. Earth Obs. Remote Sens., vol. 8, no. 3, pp. 980-993, 2015.

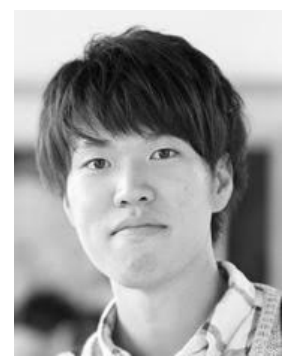

Yuta Izumi received the B.Eng., and M.Eng. degree from Chiba University, Japan, in 2016, and 2018, respectively. He is currently pursuing a $\mathrm{Ph} . \mathrm{D}$. degree with the Tohoku University, Japan. His research interests include polarimetric radar calibration, soil moisture retrieval, atmospheric phase screen compensation, and polarimetric interferometric analysis for both spaceborne and ground-based SAR. He was a recipient of the President Award of Chiba University and the Dean Award of the Graduate School of Advanced Integration Science, Chiba University, in 2018.

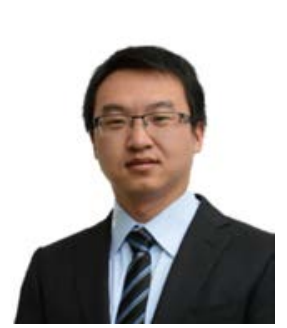

Lilong Zou (M'18) received the B.E. and M.E. degree from College of GeoExploration Science and Technology, Jilin University, Changchun, China, in 2009 and 2012, respectively. He received the Ph.D. degree in Environmental Studies from Tohoku University, Sendai, Japan, in 2016. From 2016 to 2018, he was an assistant professor at Tohoku University, Japan. Then he became a researcher at National Institute of Advanced Industrial Science and Technology (AIST), Japan. He is currently a Research Fellow with School of Computing and Engineering, University of West London, London, U.K. His research interests include the development of new algorithms, methodologies, and signal processing technologies of microwave radar systems and their applications for disaster mitigation and non-destructive testing.

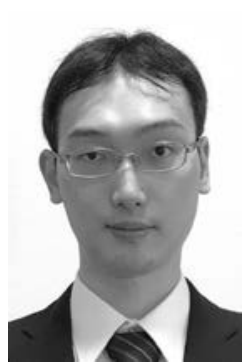

Kazutaka Kikuta received the B.Eng., M.Eng. and Ph.D. degrees from The University of Tokyo, Japan, in 2012, 2014 and 2017, respectively. Since 2017, he has been an Assistant Professor with the Center for Northeast Asian Studies, Tohoku University. His research interests include signal processing of ground penetrating radar and ground-based synthetic aperture radar. Dr. Kikuta is a Member of the IEICE.

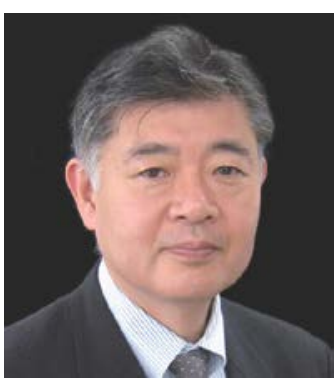

Motoyuki Sato (S'79-M'80SM'02-F'10) received the B.E., M.E degrees, and Dr. Eng. degree in information engineering from the Tohoku University, Sendai, Japan, in 1980, 1982 and 1985, respectively. Since 1997 he is a professor at Tohoku University and a distinguished professor of Tohoku University 2007-2011, director of Center for Northeast Asian Studies, Tohoku University 20092013. He was a visiting researcher at the Federal German Institute for Geoscience and Natural Resources (BGR) in Hannover, Germany in 1988-1989. His current interests include transient electromagnetics and antennas, radar polarimetry, ground penetrating radar (GPR), borehole radar, electromagnetic induction sensing, interferometric and polarimetric SAR. He has conducted the development of GPR sensors for humanitarian demining, and his sensor ALIS which is a hand-held dual sensor, has detected more than 80 mines in mine fields in Cambodia. He received 2014 Frank Frischknecht Leadership Award from SEG for his contribution to his sustained and important contributions to near-surface geophysics in the field of ground-penetrating radar. He received IEICE Best paper award (Kiyasu Award) in 2017, Achievement Award in 2019, IEEE GRSS Education Award in 2012 and IEEE Ulrich L. Rohde Innovative Conference Paper Awards on Antenna Measurements and Applications in 2017. He is a visiting Professor at Jilin University, China, Delft University of Technology, The Netherlands, and Mongolian University of Science and Technology. Dr. Sato was a member of the IEEE GRSS AdCom (2006-2014). He is an associate editor of IEEE Geoscience and Remote Sensing Letters, and a guest editor of the special issue of GPR2006 and GPR2010 in Transactions on Geoscience and Remote Sensing, and IGARSS2011, GPR2012 and GPR2014 in IEEE Journal of Selected Topics in Applied Erath Observations and Remote Sensing. He was the chair of the IEEE GRSS Japan Chapter (2006-2007). He served the general chair of IGARSS2011 and the technical chair of GPR1996. 